\title{
La protection judiciaire des droits sociaux en Colombie. Analyse jurisprudentielle.
}

\section{La protección judicial de los derechos sociales en Colombia. Análisis jurisprudencial}

Recibido: 09/02/2016 Aprobado: 30/05/2016

DOI: http://dx.doi.org/10.25054/16576799.1285

Germán Alfonso López Daza

Professeur et Directeur du Master en Droit Public

Université Surcolombiana - Colombie germanlo@usco.edu.co

Carlos Fernando Gómez García

Professeur de l'Université Surcolombiana - Colombie

carlos.gomez@usco.edu.co

Katherin Torres Posada

Professeur de l'Université Surcolombiana - Colombie katherin.torres@usco.edu.co

\section{RÉSUMÉ}

Cet article est le résultat de recherches menées par le groupe de recherche Nouvelles Visions du droit de la Faculté de droit de l'Université Surcolombiana. Ce document analyse les contextes dogmatiques des droits sociaux à travers la jurisprudence de la Cour Constitutionnelle colombienne. Le développement judiciaire des droits sociaux a été très important à tel point qu'ils ont un caractère de fondamentale.

\section{MOTS CLES}

Actions constitutionnels; Cour Constitutionnelle colombienne; Droits sociaux; Justiciabilité de droits.

\section{RESUMEN}

Este artículo es el resultado de una investigación desarrollada por el grupo Nuevas Visiones del Derecho de la Facultad de Ciencias Jurídicas y Políticas de la Universidad Surcolombiana. En este documento se realiza una presentación de los contextos tanto dogmático, así como jurisprudenciales de los derecho sociales en Colombia, que a partir de la Constitución Política de 1991, tienen carácter constitucional y que ha sido la Corte Constitucional desde sus sentencias, donde les ha dado la calidad de fundamental.

\section{PALABRAS CLAVES}

Acciones constitucionales; Derechos sociales; Corte Constitucional Colombiana; Justiciabilidad de los derechos. 


\section{INTRODUCTION}

Dans l'ancienne Constitution colombienne de 1886, les droits sociaux n'étaient pas consacrés. Cette constitution fut changée par la Charte de 1991, laquelle inclut les principaux droits sociaux tels que la santé, la sécurité sociale, l'éducation, le logement et l'eau potable. Les droits sociaux ne sont pas définis par la Constitution de 1991 mais la Cour Constitutionnelle les a développés amplement.

La Cour a dit qu'ils sont des droits fondamentaux, c'est-à-dire, droits subjectifs avec un haut degré d'importance. Mais ce qui distingue les droits sociaux d'un autre droit, c'est le caractère de droits de prestation ; à savoir, que ce sont des droits généraux positifs qui obligent l'État à donner des prestations aux citoyens.

Les droits sociaux ont été protégés par la Cour Constitutionnelle depuis 1992. C'est-à-dire, la justiciabilité des droits sociaux en Colombie a été possible grâce à l'interprétation jurisprudentielle de la Cour Constitutionnelle.

Aujourd'hui tous les juges colombiens protègent les droits sociaux au moyen l'action de "tutelle » ou action de protection de droits fondamentaux. Cette intervention des juges a été initialement critiquée parce que les juges ordonnèrent dépense de ressources publiques pour protéger les droits sociaux. Dans beaucoup cas, les juges ordonnèrent l'arrestation des fonctionnaires pour ne pas exécuter l'ordre de protection d'un droit social.

C'est pourquoi tous les juges colombiens ont dû apprendre la théorie des droits fondamentaux et droits sociaux. Ils doivent résoudre les actions de tutelle et protéger les droits fondamentaux avec les précédents jurisprudentiels de la Cour Constitutionnelle.

Les droits fondamentaux sont ces droits qui détient l'homme par le simple fait d'être, à savoir qui appartiennent à l'être humain, sans distinction de race, de statut, de sexe ou de religion. II a été donné plusieurs dénominations telles que droits de l'homme ou droits de la personne (Alexy, 2002, p. 63). Ces droits sont garantis aux citoyens où le système juridique et politique est orienté vers le respect et la promotion de la personne humaine.

Les droits fondamentaux contiennent un support solide axiologique et proviennent d'un fondement éthique en faveur de la dignité humaine. Ces droits sont appelés Droits de première génération parce qu'ils sont historiquement les premiers à atteindre une reconnaissance formelle et la protection par la législation positive. Répond à l'idéologie de la fin du XVIIle siècle et au début du XIXe siècle (Landa, 2002, p. 58). Dans ce contexte les droits civils et politiques reçurent le nom de Droits de la première génération des droits de l'homme.

Les droits de deuxième génération sont le résultat des changements produits par la révolution industrielle et de la technologie. Ils reflètent la nécessité d'intervention de l'État dans la défense des droits à l'éducation, la santé, la liberté d'association, de la culture et d'autres activités d'importance similaire (Vasak, 2004, p. 14).

Leur apparition est liée à la montée de ce qu'on appelle le constitutionnalisme social, répondre aux plexus idéologiques du début du XXe siècle. Son objectif est de résoudre le problème de la question sociale, soit répond à la préoccupation de supprimer les obstacles à caractère économique et social qui entravent le plein épanouissement de la personne humaine.

Ils se réfèrent à l'existence de conditions de vie et l'accès aux biens matériels et culturels nécessaires à la dignité de la famille humaine. La réalisation de ces droits ne dépend pas, en général, la création d'un ordre juridique ou une simple décision politique des instances gouvernementales, mais la conquête d'un ordre social en vertu de la règle de la juste répartition des biens, ce qui peut être réalisé progressivement, parce que son application est subordonnée à la disponibilité des ressources appropriées à leur satisfaction, de sorte que l'Etat acquiert l'obligation 
acquiert l'obligation de répondre aux besoins du peuple.

L'accomplissement de ces obligations implique une sorte de jugement sur la politique économique et sociale de l'État, ce qui échappe à la sphère judiciaire et est confiée à plusieurs institutions - politiques techniques.

Certains doctrinants ${ }^{1}$ ont fixé quelques caractéristiques des droits sociaux : l'existence d'une norme, une obligation et une position juridique, un niveau d'importance et un caractère général positif. Une autre caractéristique des droits sociaux est qu'ils ne peuvent être théoriquement réclamés par la voie judiciaire, puisqu'ils dépendent directement de la volonté des politiques qui définissent le développement des divers domaines d'application de ces lois, en augmentant ou en baissant les budgets respectifs. Cependant, en Colombie la Cour Constitutionnelle a ouvert le chemin vers la protection judiciaire des droits sociaux. Initialement ces décisions ont provoqué la protestation et l'opposition du gouvernement. Néanmoins, aujourd'hui la protection judiciaire de droits sociaux est acceptée par le Gouvernement et applaudi par le peuple.

Ce rapport vise à présenter de manière schématique l'évolution de la jurisprudence constitutionnelle colombienne en matière des droits sociaux. À cette fin, la question se tiendraà 7 chapitres.

Dans les premiers chapitres, on analysera le concept de développement des droits constitutionnels en Colombie et sa protection. Ensuite, on traitera la liberté de configuration législative dans le développement progressif des droits économiques, sociaux et culturels, et le développement jurisprudentiel du droit à la sante, le droit à l'éducation et le droit à l'eau. Finalement on présentera quelques conclusions.

\section{LES DROITS CONSTITUTIONNELS EN COLOMBIE}

Le concept d'Etat de droit implique la reconnaissance des droits des moins favorisés et des exclus ainsi que la recherche de la diminution des inégalités. La Cour Constitutionnelle dans son arrêt T -105 de 2009 réaffirme ce lien étroit entre la clause de l'Etat de droit et le respect de la dimension matérielle du principe d'égalité défini dans l'article 13 de la Charte colombienne (CConst. T-105/09, J.Córdoba).

Parmi les principes fondateurs de l'Etat de droit, on trouve les dispositions constitutionnelles spécifiques concernant d'abord la protection des droits dans les domaines du travail (article 54 de la CP) et de l'éducation (article 68 de la CP), et ensuite l'élaboration de politiques de prévision, de réhabilitation et d'insertion des personnes handicapées conformes aux obligations internationales de l'État (Art. $47 \mathrm{CP}$ ).

Dans un premier temps, la Cour a fait une différenciation doctrinale entre d'une part les droits civils et politiques, d'abstention et/ou de première génération, et d'autre part les droits sociaux, relatifs aux prestations et/ou de seconde génération. Les premiers sont immédiatement applicables avec obligation pour l'État de ne pas intervenir contrairement aux seconds qui requièrent des prestations positives de la part de l'État non exigibles immédiatement et nécessitant une procédure pour être garantis surtout lorsqu'il s'agit d'une prestation du service public (i) dont les ressources sont limitées (ii).

Pour ce qui est des droits sociaux, en particulier le droit à la santé, il n'y a aucune raison que les obligations négatives ou celles de caractère positif n'impliquant pas une dépense représentative (donner l'information) soient soumises à une réalisation à caractère progressif; par ailleurs, toutes les obligations positives n'ont pas un caractère progressif (ii). En effet, l'obligation d'adopter toutes

1- Par exemple, ARANGO RIVADENEIRA, Rodolfo. El concepto de derechos sociales fundamentales. Bogotá: Legis, pag. 38. 
les mesures possibles conformément aux ressources disponibles et de garantir les dispositions concrètes établies par les organes compétents pour la protection des droits sont des obligations à caractère immédiat.

La Cour a exprimé dans ses toutes premières sentences que cette distinction présente un certain intérêt académique pour la compréhension de l'origine et de la création de certains droits mais qu'elle n'a en aucun cas une valeur normative. Elle ne peut être invoquée pour nier de façon définitive la justiciabilité d'un groupe de droits ou l'efficacité directe d'un autre groupe. Ceci s'explique par le fait que tout droit constitutionnel présente des facettes positives ou négatives.

De la même façon que la Cour a précisé que les obligations positives n'ont pas toutes un caractère progressif, elle a affirmé que les droits civils et politiques ne sont pas tous garantis par la non intervention de l'État.

Le tribunal constitutionnel a également indiqué que le fondement d'un droit ainsi que sa justiciabilité (ou exigibilité par voie judiciaire) ne sont pas logiquement des questions implicites, puisque les droits fondamentaux sont toujours susceptibles d'être soumis à des limitations raisonnables et proportionnées imposées afin de respecter d'autres principes constitutionnels. En conclusion, toutes les prestations correspondant à la protection d'un droit ne peuvent être exigées par voie judiciaire et toutes les particularités mentionnées peuvent s'expliquer par la complexité des droits constitutionnels.

Les obligations de respect, protection, garantie ou exécution du droit concernent plusieurs autorités qui les appliquent de différentes façons dans le temps. Ces considérations ont eu une influence considérable sur le développement de la jurisprudence constitutionnelle dans le domaine de la santé comme nous le verrons maintenant.

\section{LA PROTECTION DES DROITS SOCIAUX, ECONOMIQUES ET CULTURELS PAR L'ACTION DE TUTELLE}

La doctrine constitutionnelle a longtemps fait une distinction théorique entre les droits civiques et politiques faisant l'objet d'une protection judiciaire directe appelée Action de tutelle et les droits sociaux, économiques et culturels défendus par une procédure législative ou administrative.

Cependant, la Cour constitutionnelle a admis dans ses premiers arrêts, que l'action de tutelle peut également protéger des droits de la deuxième catégorie qui soient étroitement liés à un droit fondamental, position de la Cour connue comme «thèse de la connexité ». La jurisprudence de la Cour ya recours pour défendre le droit à la santé.

«Un droit fondamental par connexité est un droit qui n'apparaît pas en tant que tel dans la constitution, chapitre 1 , paragraphe 2 , relatif aux droits constitutionnels fondamentaux mais qui est considéré comme tel en vertu de son lien intime et incontestable avec ceux-ci. Si un droit non défendu menace un droit fondamental, il est alors considéré comme droit fondamental par connexité. C'est le cas du droit à la santé, non considéré comme fondamental, mais qui le devient au cas où la non-assistance du patient met en danger son droit à la vie et bouleverse l'intégrité de la personne et la sécurité sociale de l'affilié ; il peut par conséquent être protégé par une action de tutelle.» (CConst. T-398/96, J. Arango)

La classification en chapitres correspondant à la division en catégories de droits fondamentaux, ignore la notion même de droits de l'homme en tant que droits humains inhérents (peu importe son apparence dans le temps et classement), qui sont fondés sur la dignité de son statut en tant que personne et les rend essentiellement liés, interdépendants et indivisibles.

La seconde étape de la protection des droits économiques, sociaux et culturels se fait à partir de l'interprétation de la jurisprudence antérieure dans l'arrêt SU-819 de 1999, selon «le critère de la 
transmutation». Bien que ces droits soient de caractère prestationnel, puisque leur application dépende de normes budgétaires et de procédures, ils ont tendance à se transformer en droit subjectif du fait même qu'il y a des éléments concrets qui permettent d'exiger de l'Etat d'exécuter une prestation dont il est redevable. Ainsi, le devoir d'assistance s'exerce à faveur du plaignant.

Postérieurement, la Cour, dans l' arrêt T-197 de 2014, a précisé que les droits civils et politiques ainsi que les droits sociaux, économiques et culturels sont des droits fondamentaux impliquant des obligations à caractère aussi bien négatif que positif.

L'État est dans l'obligation de respecter et d'appliquer ces droits à caractère de prestation. Dans ce sens, tous les droits fondamentaux, quels qu'ils soient, présentent tous un caractère à prestation et dépendent donc des décisions budgétaires prises par l'État.

II serait contradictoire de ne pas considérer les droits à caractère de prestation, le droit à la santé, à l'éducation, au logement, à l'eau potable comme des droits fondamentaux et aucun droit, y compris celui à la vie, ne pourrait avoir le caractère de fondamentalité.

De plus, ce serait aller à l'encontre des exigences internationales en matière de droits humains acceptées par l'État colombien et de l'article 93 de la Charte politique du pays.

La Cour Constitutionnelle (arrêt T-197, 2014) a tenu compte de la sentence de la Cour Interaméricaine des droits humains dans le cas Acevedo Buendia y otros Vs.Perú non pas pour établir des obligations en matière de droits sociaux, économiques et culturels, mais pour en dégager l'herméneutique en matière de protection de ces droits dans le système interaméricain: "Dans ce sens, la Cour considère qu'il est pertinent de rappeler l'interdépendance entre les droits civils et politiques et les droits sociaux, économiques et culturels, ces derniers devant être considérés comme des droits humains, non hiérarchisés et exigibles auprès des autorités compétentes ».

Selon la ligne jurisprudentielle de la Cour constitutionnelle, la fondamentalité des droits ne dépend pas et ne peut dépendre de la façon dont ils sont appliqués dans la pratique. Tous les droits sont fondamentaux puisqu'ils protègent directement les valeurs que les constituants ont voulu inclure démocratiquement dans la catégorie des biens protégés par la Constitution.

Ces valeurs sont transcrites en normes juridiques qui définissent les frontières matérielles qui sont une protection contre l'arbitraire de l'État.

Ce qui revient à dire que dans un état démocratique et social de droit, tous les individus n'ont pas les mêmes chances et les mêmes opportunités et que c'est l'État qui doit pallier à ce déséquilibre en protégeant les personnes les plus démunies.

Le caractère fondamental des droits étant défini, il reste à considérer l'aspect concret, à savoir les actions qui protègent ces droits. Dans un pays comme la Colombie, caractérisé par un manque de ressources, c'est l'État qui doit garantir les droits et la dignité des personnes les plus défavorisées.

Aujourd'hui n'a pas du sens l'existence des exigences procédurales pour l'action de tutelle, par exemple au moyen de création judiciaire de la connexité d'un droit de première génération aven un droit de deuxième génération. C'est pourquoi la Cour a dit que tous les droits sont fondamentaux (CConst. T-016/07, H. Sierra).

\section{LA FONDAMENTALISATION DES DROITS SOCIAUX}

La jurisprudence de la Cour constitutionnelle a admis que les droits civils et politiques ainsi que les droits sociaux, économiques et culturels sont des droits fondamentaux qui impliquent des obligations négatives et positives. 
La Cour Constitutionnelle colombienne s'est inspirée de la doctrine internationale élaborée depuis la naissance des droits de l'homme pour réfléchir d'abord sur la différenciation faite par les constituants de 1991 entre les droits fondamentaux et les droits sociaux, économiques et culturels dits de seconde génération et à caractère prestationnel, et ensuite sur la justiciabilité et le caractère fondamental de ces derniers.

Dans un premier temps, elle a adopté la division stricte entre droits sociaux et fondamentaux et par conséquent admis l'absence de protection judiciaire des premiers. Dans un deuxième temps, elle a accepté de les qualifier de « fondamentaux » en vertu de leur relation incontestable avec les autres droits fondamentaux, qui pourraient être menacés si les droits sociaux n'étaient pas respectés.

Grâce à la «thèse de connexité», elle a autorisé la protection constitutionnelle des droits à la propriété, aux loisirs, à la protection de l'environnement, à la santé, au logement lorsque leur non application mettait en danger des individus étant tout particulièrement protégés par la constitution ou menaçait le droit à l'égalité, à la vie, à la dignité du plaignant.

Dans un troisième temps, le "critère de transmutation» a permis de résoudre le dilemme sur l'exigibilité des prestations découlant des droits sociaux et d'obliger l'État à exécuter ses obligations.

Elle a établi que les droits sociaux pouvaient acquérir des conditions d'effectivité et donc être protégés par action de tutelle, lorsque l'obligation de l'État serait démontrée de façon normative.

Les arguments relatifs au caractère "fondamental» des droits sociaux ont été réaffirmés par la jurisprudence actuelle pour que l'État respecte ses engagements dans ce domaine. Elle a rappelé que:

i) Le caractère de droit «fondamental» répond à plusieurs critères, le plus important étant celui de la dignité humaine ; ii) tous les droits fondamentaux, aussi bien ceux de première que de deuxième génération, sont positifs et négatifs, et par conséquent dépendent des distributions budgétaires de l'État ; iii) bien qu'en étroite relation, le caractère "fondamental» et la justiciabilité des droits sociaux se réfèrent à des notions différents: le premier se rapporte aux valeurs que les constituants ont voulu protéger en les incluant dans la constitution et la seconde à la possibilité d'exiger le respect des droits par voie judiciaire (CConst. T-743/13, L. Vargas).

En conclusion, la Cour a déclaré que les sphères positives des droits constitutionnels, tous fondamentaux, sont exigibles par voie judiciaire en fonction de l'importance que leur accorde la charte politique, si d'une part ils ne requièrent pas un effort financier trop élevé de la part de l'État et si leur exécution est une garantie à la dignité humaine telle que la définit sa jurisprudence ou le droit international dans le domaine des droits de l'homme.

Le côté pratique de cette position de la cour est immédiat : le juge ne doit plus désormais démontrer le caractère fondamental du droit social à prestation en cas d'action de tutelle réclamant la protection d'un droit fondamental menacé.

Sa tâche consistera à vérifier quelles sont les obligations de l'État, et si la demande est justiciable d'un point de vue national et international.

L'État ne doit pas méconnaître ces droits et a obligation de mettre en œuvre une politique tenant compte de ses devoirs prestationnels.

Tous les droits constitutionnels fondamentaux, civils, politiques, sociaux, économiques, culturels, environnementaux, impliquent un caractère prestationnel et doivent faire l'objet d'une prévision budgétaire afin d'être garantis (CConst. T-160/11, H. Sierra).

II serait contradictoire de ne pas reconnaître les droits sociaux comme étant fondamentaux et selon cette position on arriverait à la conclusion absurde que le droit à la vie ne le serait pas non plus. 
D'autre part, la non reconnaissance de la fondamentalité des droits sociaux reviendrait à ignorer les traités internationaux relatifs aux droits de I'homme définis par l'article 93 de la Constitution'.

Selon la jurisprudence de la Cour, le caractère fondamental des droits sociaux ne dépend en aucun cas de leur effectivité. Ils ne peuvent être conditionnés à l'existence d'une loi qui les mettrait en œuvre.

Tous les droits sont fondamentaux, puisqu'ils ont tous un lien étroit avec les valeurs défendues par la Constitution par des normes juridiques que l'État doit respecter sous peine d'agir arbitrairement.

Il en découle que dans un État social et démocratique de droit, tous les individus n'ont pas les mêmes chances et opportunités de vivre dignement.

C'est donc l'État qui doit protéger les personnes démunies et fragiles. C'est à l'État de corriger les déséquilibres et inégalités sociales existant dès la naissance.

Une fois, la fondamentalité des droits sociaux étant reconnue, il reste à examiner leur effectivité. Comment sont-ils défendus dans la pratique? Quelles actions judiciaires sont prévues pour les faire respecter? Dans un pays comme la Colombie dont les ressources sont insuffisantes, c'est à l'État et à ses représentants que revient le rôle de veiller au respect des principes d'équité, de solidarité, d'assistance et de mener des politiques qui garantissent les droits sociaux aux personnes les plus démunies et de leur donner les moyens d'accéder à une vie digne.

Par exemple, dans le cas du droit fondamental à la santé, la Cour a dit à plusieurs reprises que ce n'est pas un droit dont la protection peut être demandée prima facie par action de tutelle. Son caractère prestationnel oblige l'État à prévoir un budget couvrant une garantie totale de ce droit ainsi que tous les autres dans un contexte financier insuffisant.

La recevabilité de l'action de tutelle ne peut aujourd'hui se baser sur la connexité avec les droits fondamentaux puisque tous ont plus ou moins un caractère prestationnel indéniable, mais sur l'ensemble des circonstances qui obligent à ce recours judiciaire pour que le droit fondamental soit effectif.

\section{LIBERTÉ DE CONFIGURATION LÉGISLATIVES DANS LE DÉVELOPPEMENT PROGRESSIF DES DROITS ÉCONOMIQUES, SOCIAUX ET CULTURELS}

La compétence du législateur dans l'élaboration et développement progressif des droits sociaux et culturels est définie dans la Constitution colombienne de 1991. L'article 152 de la Charte consacre les lois statutaires lesquels doivent développer les droits fondamentaux et les moyens de protection ${ }^{3}$.

La jurisprudence constitutionnelle a expliqué que «la Constitution donna au Congrès une grande liberté

2 - Article 93. Les traités et conventions internationaux ratifiés par le Congrès qui reconnaissent les droits de l'homme et interdisent leur limitation dans l'état d'urgence prévalent dans l'ordre interne. Les droits et obligations mentionnés dans la présente Charte sont interprétés conformément aux traités internationaux relatifs aux droits de l'homme ratifiés par la Colombie. L'État colombien peut reconnaître la compétence de la Cour pénale internationale dans les conditions prévues par le Statut de Rome adopté le 17 Juillet 1998 par la Conférence de plénipotentiaires des Nations Unies et par conséquent de ratifier ce traité conformément à la procédure prévue dans la présente Constitution. L'admission d'un traitement différent sur les questions de fond par le Statut de Rome en ce qui concerne les garanties contenues dans la Constitution ne prendra effet que dans le cadre de l'affaire réglée dans le Statut.

3 - Article 152. À travers les lois statutaires, le Congrès réglera les questions suivantes:

a) Les droits et devoirs des individus et les procédures et les recours pour leur protection fondamentales;

b) L'administration de la justice;

c) L'organisation et système de partis et mouvements politiques; statut de l'opposition et les fonctions électorales;

d) Les institutions et les mécanismes de participation des citoyens.

e) Les états d'urgence.

f) L'égalité électorale entre les candidats à la présidence de la République qui remplissent les conditions fixées par la loi. 
pour définir la portée de ces droits et des mécanismes institutionnels concrets et des procédures pour la mise en œuvre efficace» ${ }^{4}$.

Toutefois, cela ne signifie pas que le développement des droits prestationnels peuvent être ignorés 0 méconnaitre les valeurs constitutionnelles ni la base juridique constitutionnel relatifs aux droits de l'homme ratifiés par le Congrès, qui engage le gouvernement colombien avec le la mise en œuvre progressive des droits sociaux et culturels.

Par conséquent, la Cour constitutionnelle dans l'arrêt C-1165 de 2000, a déclarée inconstitutionnelle l'article 34 de la loi 344 de 1996. Cette décision a établi que la baisse des ressources pour le système de santés subventionnées, en aucune façon tient fondement dans le postulat constitutionnel sur l'extension progressive de la sécurité sociale et la couverture, d'après l'article 48 de la Charte.

Il est évident que moins de ressources et plus des besoins non satisfaits dans la santé, étant donné les circonstances socio-économiques dans le pays, plutôt que d'augmenter la couverture de la sécurité sociale et la qualité de service, une réduction des ressources affectera négativement la population au détriment du bien-être de ceux qui ont le plus besoin de la solidarité tels que le personnes pauvres, diminué ou en état de chômage (CConst.T1165/2000).

La Cour a dit que la rationalisation des dépenses publiques ne justifie pas la diminution aussi drastique des contributions du budget national destiné aux besoins de santé des secteurs les plus pauvres de la population colombienne, en particulier lorsque l'on considère la couverture de la sécurité sociale encore très loin de couvrir l'ensemble de la population colombienne.

Dans le même sens, la sentence C-671 de 2002 la Cour a déclaré que sans préjudice de l'objectif légitime de protéger la viabilité financière des régimes spéciaux pour la santé de la sécurité sociale, la réduction de l'ensemble des bénéficiaires, l'exclusion est lui-même « un revers pour assurer le droit à la santé, des gens qui avaient garanti la protection de ce droit constitutionnel, sont alors exclus du système sans aucune garantie de couverture $»$.

La Constitution et les traités des droits humains disent que tous les droits sociaux ne sont pas immédiats et la mise en œuvre complète, toutefois, les États ont non seulement le devoir de prendre toutes les mesures possibles pour obtenir la réalisation progressive intégrante, mais doit également veiller à ce la jouissance de ces droits à tous les citoyens sans discrimination. Par conséquent, comme la Cour l'a déjà expliqué, en harmonie avec la jurisprudence et la doctrine internationale sur le sujet, le mandat de l'escalade ne devrait pas être traité comme une justification de l'inaction de l'Etat dans la protection de ces droits.

Par contre, l'État colombien a pris des engagements internationaux et constitutionnels clairs par rapport aux prestations des droits sociaux tels que la santé, l'éducation et de l'eau.

D'une part, l'État tient l'obligation de commencer immédiatement le processus menant à la pleine réalisation des droits sociaux. Et d'autre part, il y a des contenus minimaux ou essentiels pour la satisfaction du droit que l'Etat doit garantir à toutes les personnes. Autrement dit, la progressivité fait référence à la reconnaissance de meilleures prestations par rapport à chacun de ces droits sociaux, mais ce mandat ne dispense pas la violation du devoir de l'Etat d'assurer, dès que possible, la couverture universelle du contenu minimal de ces droits, comme la Cour l'a déjà reconnu.

Cet ordre de progressivité implique qu'une fois atteint un certain niveau de protection, la grande liberté du législateur dans la définition des droits sociaux est diminuée, au moins sur un point: le recul du niveau de 
protection obtenu devrait être présumé, en principe, inconstitutionnelle et il est donc soumis à un contrôle judiciaire strict. Afin d'être constitutionnelle, les autorités doivent démontrer qu'il y a des raisons impérieuses qui font qu'il est nécessaire que l'étape régressive dans le développement d'un droit social de performance basé (CConst. C-228/11, J. Henao).

\section{DEVELOPPEMENT JURISPRUDENTIEL DUDROIT ALASANTE}

L'article 49 de la Constitution stipule que le droit à la santé est un droit à la fois constitutionnel et un service public. Ce qui veut dire que tous les individus ont droit à la santé et que l'État a l'obligation de respecter, protéger et d'adopter des mesures pour assurer la réalisation de ce droit conformément aux principes d'efficacité, d'universalité et de solidarité (CConst. T160/11, H. Sierra).

Cet article précise que l'État doit mettre en œuvre les politiques de prestation du service de santé fournies par les entreprises privées et exercer un contrôle sur ces plans d'action.

Cette protection du droit à la santé est renforcée par le droit international. L'article 25 de la déclaration universelle des droits de l'homme dit dans le paragraphe 1:

Toute personne a droit à une vie digne qui lui assure à lui et à sa famille l'accès à la santé, soit à l'alimentation, I'habillement, l'assistance médicale et les services sociaux.

L'article 12 du Pacte international relatif aux droits économiques, sociaux et culturels énonce très clairement et de façon exhaustive les dispositions relatives au droit à la santé : le paragraphe 1 dispose que les États parties reconnaissent « Le droit de tout individu à jouir d'une santé mentale et physique optimale». Le paragraphe 2 énumère à titre d'exemples les mesures que les États doivent adopter en vue d'assurer la pleine réalisation de ce droit.
L'Observation générale 14 du Comité des Nations Unies relative aux droits sociaux, économiques et culturels rappelle que:

"La santé est un droit fondamental nécessaire ; sa réalisation est indispensable à la jouissance d'autres droits de l'homme. Tout être humain a le droit à jouir du meilleur état de santé possible.» (CConst. T160/11, H. Sierra)

La Cour a argumenté la fondamentalité du droit à la santé en s'appuyant sur les décisions suivantes:

a) L'adoption de ce critère par la Salle plénière (arrêt C-811, 2007), dans le cas des couples homosexuels où l'un des individus est exclu du système de sécurité sociale et ne peut être ayant-droit de son compagnon ou compagne. Ce qui équivaut à un déficit de protection inacceptable d'un point de vue constitutionnel ; et

b) La doctrine du droit international des droits de l'homme qui prône l'égalité, l'interdépendance et l'indivisibilité de tous les droits.

La jurisprudence constitutionnelle définit le droit à la santé comme la faculté d'un individu à maintenir un fonctionnement organique normal aussi bien physiquement que mentalement et de se rétablir en cas de trouble organique et fonctionnel.

La Cour a également repris les quatre éléments qui protégeaient ce droit conformément à l'Observation générale 14 du Comité des droits sociaux, économiques et culturels:

b) L'accessibilité: les établissements, biens et services de santé doivent être accessibles à tous, dans la juridiction de l'État partie. Cette accessibilité inclut quatre dimensions:

i) la non-discrimination : les établissements, biens et services de santé doivent être accessibles aux populations les plus vulnérables et les plus marginalisées, sans aucune discrimination. 
ii) accessibilité physique : cela implique une proximité géographique des établissements de santé pour tous les groupes sociaux vulnérables ou marginalisés, ainsi que pour les minorités ethniques, populations indiennes, femmes, enfants, adolescents, personnes âgées, handicapés, personnes atteintes du SIDA ,l'accès aux services médicaux, à l'eau potable, à des toilettes pour tous , y compris dans les zones rurales, ainsi que des accès aménagés pour les personnes handicapées.

iii) accessibilité économique : il doit y avoir équité d'accès aux soins. Tous les services de santé et autres fournis par des établissements publics ou privés doivent obéir à ce principe d'équité qui exige une charge financière proportionnelle aux revenus des foyers (CConst. T-105/08, J. Córdoba).

\section{LE DROIT A L'EDUCATION, DROIT SOCIAL FONDAMENTAL}

L'article 67 de la Constitution Politique colombienne reconnaît que l'éducation est à la fois un droit et un service public devant garantir aux citoyens l'accès à la connaissance, à la science et à la culture ${ }^{5}$.

Cet article renforce la protection du droit à l'éducation pour les enfants, énoncé dans l'article 44 de la Constitution et souligne son importance pour la connaissance, le développement de la personnalité, l'égalité des chances et l'accès à la culture.

La norme supérieure a assigné la coresponsabilité de cette mission sociale d'envergure à la famille, la société et l'État. Elle a obligé l'État à mettre en œuvre des actions afin de réguler et contrôler le service de l'éducation et d'en garantir la qualité et la couverture géographique.
En tant que service public, l'éducation de l'Etat nécessite une action spécifique, liée à leur assurer une prestation efficace et continu à tous les habitants du pays, dans le respect des principes d'universalité, de solidarité et de redistribution des ressources dans la population économiquement vulnérables.

Dans sa dimension de droit, l'éducation a le caractère fondamental en ce qui concerne le rôle qu'il joue dans la promotion du développement humain et l'éradication de la pauvreté. Aussi à cause de son impact sur la réalisation des autres droits fondamentaux comme la dignité humaine, l'égalité des chances, le minimum de subsistance, la liberté de choisir une profession et la participation politique.

La Cour Constitutionnelle colombienne a reconnu dès ses premiers arrêts de la nature complexe de l'éducation en alignant son service public et de droit double. Néanmoins, considéré, d'une lecture exégétique de l'article 67 de la C.P., que l'accès et la permanence dans le système d'éducation font partie de son noyau essentiel du droit et, après avoir été appelé par la Charte comme un droit fondamental des enfants, la prestation est obligatoire jusqu'à 18 ans.

Les paramètres définis par le Comité, qui es l'interprète autorisé du Pacte international relatif aux droits économiques, sociaux et culturels (PIDESC), dans l'Observation générale $n^{\circ} 13$ sur le contenu normatif de l'article 13 du Pacte, sur les buts de l'éducation, transformé cette conception diamétralement.

Depuis lors, la jurisprudence constitutionnelle a reconnu que le droit à l'éducation comporte quatre composants interdépendants structurels qui, à leur tour, sont réalisées par trois types d'obligations

5 - Article 67. L'éducation est un droit de l'individu et un service public qui a une fonction sociale; l'éducation a pour but l'accès à la connaissance, la science, la technologie, et d'autres biens et valeurs de la culture. L'éducation doit former sur le respect des droits de I'homme, de la paix et de la démocratie; et dans la pratique du travail et de loisirs, pour l'amélioration culturelle, scientifique, technologique et protection de l'environnement. L'État, la société et la famille sont responsables de l'éducation, qui est obligatoire entre cinq et quinze ans et doit comprendre au moins un an d'âge préscolaire et neuf ans du lycée. L'enseignement est gratuit dans les institutions publiques, sous réserve du paiement des frais de scolarité à ceux qui peuvent se permettre. Il correspond à l'état d'effectuer l'inspection finale et la supervision de l'éducation afin d'assurer leur qualité, le respect de ses objectifs et le meilleur développement moral, intellectuel et physique des apprenants; assurer une couverture adéquate du service et assurer aux enfants les conditions nécessaires à l'accès et la permanence dans le système éducatif. La nation et les entités territoriales doivent participer à la gestion, le financement et l'administration des services éducatifs de l'Etat, selon les termes de la Constitution et la loi. 
différents. Ces composants ou caractéristiques dimensionnelles seront développées brièvement cidessous.

\subsection{Disponibilité}

Ce composant se réfère à répondre à la demande éducative de deux façons: la promotion de l'offre publique et d'encourager la création d'établissements d'enseignement privés. Mais cela signifie également que ces institutions et les programmes offerts sont disponibles aux étudiants.

Cela signifie respect de certaines conditions qui peuvent varier en fonction du contexte, tels que les infrastructures, matériel d'étude, des installations sanitaires avec des salaires concurrentiels, les bibliothèques, la technologie, etc.

En conclusion, la composante de la disponibilité de l'éducation comprenant i) l'obligation de l'État de créer et de soutenir les établissements d'enseignement; ii) la liberté des individus de fonder ces établissements et iii) investir dans les ressources humaines et matérielles au service.

\subsection{Accessibilité}

L'accessibilité protège le droit individuel à entrer dans le système éducatif en conditions d'égalité ou, en d'autres termes, l'élimination de toutes les formes de discrimination qui peuvent entraver l'accès.

Plus précisément, il est considéré que ces conditions d'égalité comprennent i) l'incapacité à limiter motifs d'accès interdit, de sorte que chacun a sa place, en particulier ceux qui font partie des groupes les plus vulnérables; ii) l'accessibilité géographique ou physique, réalisé avec les institutions d'accès raisonnables et des outils technologiques modernes et iii) l'abordabilité, impliquant l'enseignement primaire gratuit et l'introduction progressive de l'enseignement secondaire et supérieur gratuit.

\subsection{Adaptabilité}

L'exigence d'adaptabilité récuse l'idée que les étudiants qui doivent satisfaire aux conditions de fourniture de services éducatifs en vigueur dans chaque établissement, et nécessite, en revanche, il est le système qui répond aux besoins des étudiants, évaluer le contexte social et culturel dans lequel ils opèrent, afin d'éviter le décrochage scolaire.

Pour cette raison, la composante de la satisfaction de l'adaptabilité a été liée à des mesures pour adapter l'infrastructure des institutions et des programmes d'apprentissage pour les conditions requises par les étudiants, en particulier ceux qui font partie de groupes de population une protection spéciale, comme les personnes handicapées ou les capacités intellectuelles exceptionnelles, les enfants qui travaillent, les enfants qui sont privés de leur liberté, les étudiants issus de groupes minoritaires ethniques, les femmes qui sont enceintes et les étudiants résidant dans les zones rurales. Le composant spécifique d'aspiration de l'adaptabilité est, en définitive, pour que les étudiants restent dans le système éducatif.

\subsection{Acceptabilité}

Observation générale $n^{\circ} 13$ du Comité Interprète du PIDESC (Comité des DESC) exige que la forme et le contenu de l'enseignement, y compris les programmes et méthodes d'enseignement, soient acceptables. Cela implique la pertinence, culturellement appropriés et de bonne qualité. Aussi, pour répondre aux objectifs de l'éducation mentionnés à l'article 13 du Pacte et les normes approuvés par chaque État en matière d'éducation. En outre, l'acceptabilité de l'enseignement implique l'équité.

Finalement, la Cour estime important de préciser que la conformité de l'acceptabilité de la part de l'assurance de la qualité de l'enseignement devrait être examinée dans le cadre du consensus auquel chaque pays met en œuvre par rapport aux priorités en matière d'éducation. 
Dans son troisième rapport d'étape au Programme d'éducation pour tous, I'UNESCO a abordé cette situation dans les termes suivants:

«Bien que des tentatives pour définir «éducation de qualité» soulève une controverse considérable, dans ce troisième rapport de suivi sur l'EPT est évident qu'il y a des bases solides pour une compréhension commune. La question de la qualité doit être considérée compte tenu de la façon dont les différentes sociétés définissent le but de l'éducation. En plus de ces deux objectifs principaux: le premier est d'assurer le développe-ment cognitif des élèves; la deuxième de souligner que l'éducation encourage le développement créatif et affectif afin qu'ils puissent acquérir des valeurs et des attitudes qui leur permettent d'être des citoyens responsables. Enfin, la qualité doit passer le test de l'équité comme un système d'éducation qui est discriminatoire à l'encontre d'un groupe particulier, que ce soit, ne remplit pas sa mission.» (CConst. T-743/13, L. Vargas)

Le devoir de l'Etat de réglementer les normes minimales régissant la fourniture des services éducatifs, est importante pour vérifier l'acceptabilité éducative dans le cadre de la qualité dans un cas particulier.

Fondée ces hypothèses de base, la tâche de l'Etat est composé d'assurer leur pleine conformité avec le principe de progressivité el l'interdiction de la régressivité de la couverture des droits sociaux, tels que l'éducation.

En outre, les instruments internationaux ratifiés par la Colombie ont considéré que le concept d'éducation acceptable implique: un contrôle adéquat et le suivi de l'activité éducative, l'interdiction des châtiments corporels et les traitements humiliants ou dégradants, l'adoption de mesures pour assurer que l'éducation est vue culturel aux minorités et de la formation des enseignants.

\section{LA PROTECTION DU DROIT À L'EAU PAR L'ACTION DE PROTECTION DE DROITS FONDAMENTAUX}

La Cour constitutionnelle colombienne a porté sur l'étude sur la viabilité de la protection judiciaire de l'eau et considérant que la nature a une double fonction de l'utilisation qui en est faite: droit fondamental et au droit collectif même temps.

La Cour estime dans les actions de tutelle si la demande de protection est visée pour protéger le droit à l'eau dans le sens général de protection de l'action de tutelle ; sinon, la personne doit présenter une action populaire consacrée dans la loi 472 de 1998 pour la protection d'un droit collectif.

La ligne jurisprudentielle sur le droit à l'eau peut être divisée en deux étapes. Dans une première étape caractérisée par le traitement timide par la Cour constitutionnelle, car il se trouve que l'eau n'a pas été traitée comme un droit fondamental, mais comme un élément essentiel pour la vie humaine élément qui peut conduire à une violation du droit à la vie, la santé, la santé publique et de la dignité.

Dans cette première période se trouve un groupe d'arrêts qui sont considérés comme représentatifs, parce qu'elles traitent l'eau comme un élément essentiel à la vie. La Cour souligne l'importance du précieux liquide. Ils sont des exemples de ce groupe d'arrêts: T-578/1992 MR Alejandro Martínez Caballero, T-244/1994 MR Hernando Herrera Vergara, T-244/1994 MR Hernando Herrera Vergara, T-092/1995 MR Hernando Herrera Vergara et T379/1995 Antonio Barrera Carbonell.

Dans ces décisions, la Cour protège le droit au service de l'eau, le droit à un environnement sain et le droit à la santé publique, entre autres, comme un moyen direct pour la protection de l'eau, mais elle ne parle pas encore de droit fondamental. Dans les deux derniers arrêts, la Cour a protégé le droit fondamental à l'approvisionnement d'eau potable pour la consommation des plaignants et a ordonné la construction d'aqueducs dans les municipalités de 
Aipe (département du Huila) et Guaduas (département du Cundi-namarca).

Dans une autre décision importante (arrêt SU442/1997), la Cour a protégé le droit à la vie et à l'approvisionnement d'eau potable. Cette décision a été prise compte tenu l'insuffisance des services publics de l'aqueduc et d'égouts du quartier du Santa Marte nommé Taganga.

La Cour a ordonné au gérant de l'Enterprise Metroagua et au maire du Santa Marta d'engager la construction d'une nouvelle station de traitement d'eau potable pour le quartier.

II est à noter qu'en dépit d'être de l'action de protection de droits collectifs l'utilisée parce qu'ils sont des droits collectifs, la Cour a protégé le droit parce que c'était une question de grand impact sur une communauté.

Dans la seconde étape, la Cour fait un développement beaucoup plus profond et direct du droit à l'eau comme un droit fondamental. Cette période peut être située entre 2007 à 2013 et se caractérise par la reconnaissance en tant que droit fondamental autonome. Cependant, les faits de l'arrêt étaient similaires de l'antérieure époque. (Accès à l'eau comme un service public).

Le but de cette protection était de garantir à une personne ou à la communauté l'accès à l'eau pour la consommation humaine, ou dans le cas où une entreprise de services publics suspendre totalement ou partiellement le service, et cette action affecte les droits fondamentaux des personnes avec protection constitutionnelle spéciale.

La Cour a dit que le droit à l'eau est une hypothèse fondamentale d'autres droits tels que le droit à la santé et le droit à l'éducation. Aussi le droit à l'eau fait partie du droit à un environnement sain, et les droits à la protection de la diversité ethnique et culturelle, étant donné que certaines communautés autochtones et afro-colombiennes ont des liens privilégiés avec la nature et l'eau.
La Cour a également protégé le droit fondamental à l'eau à partir de trois points de vue: selon les garanties minimales de la disponibilité, l'accessibilité, la qualité et la non-discrimination dans la distribution.

La disponibilité du service public à l'eau est violée dans les cas où l'accès est nié dû au manque du paiement. Exemple de ce cas est l'arrêt T-616/2010 MR Luis Ernesto Vargas Silva.

Le deuxième cas, celui de l'accessibilité est violé lorsqu'il empêche l'accès aux services nécessaires et la fourniture de services d'eau adéquats dans les cas où l'entreprise d'eau refuse d'installer ces connexions, ou quand imposent des coûts disproportionnés comme condition pour fournir l'infrastructure des réseaux locaux. Ce fut le cas de l'affaire T-279/2011 MR Luis Ernesto Vargas Silva dans lequel la Cour a reconnu le droit fondamental à l'eau potable.

Dans le troisième cas lié à l'aspect de la qualité, celui-ci est violé lorsque l'eau n'est pas reçue dans des conditions chimiques et physiques acceptables. II a été le cas décrit dans le jugement T-410/ 2003 MR Jaime Córdoba Triviño.

La Cour constitutionnelle a également établi les caractéristiques du droit fondamental à l'eau qui peutêtre spécifié comme suit:

- II présente un lien avec d'autres droits fondamentaux tels que la vie (CConst. T-567/12, M. Gonzalez)

- II peut avoir le caractère du droit fondamental ou collectif(CConst. C220/11, E. Montealegre)

- II n'est pas un droit absolu

- Le principe de l'immédiateté (CConst. T-541/13, J. Pretelt)

- II s'agit d'un service public par l'Etat (CConst. T1089/11, L. Vargas)

- Le droit a un côté positif et négatif (CConst. T749/12, M. Calle)

Enfin, la non-discrimination dans la distribution est justifiée dans les cas où il n'existe pas de 
discrimination dans la distribution, de sorte que toutes les personnes ont accès à des quantités suffisantes du liquide.

De même, dans un arrêt récent, la Cour constitutionnelle protège le droit fondamental à l'eau et de la nourriture des peuples autochtones (groupe ethnique de Dujos, Tamaz-Paez) lorsque la Cour a protégé l'accès à l'eau de la réserve indienne Paniquita. Ce groupe ethnique avait souffert la violation de leurs droits à cause de la réglementation d'une concession et la servitude d'écoulement des eaux qui traverse la réserve indienne par une entité environnementale publique.

Dans ce cas, la Cour a reconnu la violation du droit fondamental à l'eau aux peuples autochtones et à la nourriture en tant que l'entité publique empêchait l'accès àl'eau.

Cette décision de la Cour est importante parce que c'était la première fois qu'un arrêt protégeait le droit fondamental à l'eau des communautés autochtones ainsi le droit à la nourriture. La restriction d'alimentation faite par l'organisme de l'État avait empêché la pleine jouissance de l'eau et le développement des activités agricoles des communautés autochtones affectant leur droit à une alimentation adéquate.

Ces décisions jurisprudentielles de la Cour Constitutionnelle montrent son souci de protéger le droit à l'eau malgré la non-existence d'une disposition constitutionnelle spécifique qui prévoit ce droit fondamental.

En conclusion, on doit affirmer que l'eau potable est un droit fondamental reconnu par la Cour, qui fait partie du noyau dure du droit à la vie avec dignité lorsqu'ils sont destinés à la consommation humaine.

Dans le même sens, alors, la Cour a jugé que le droit à l'eau peut être protégé par la tutelle quand elle contribue à la vie, la santé et la sécurité des gens, mais ce n'est pas fondamental quand il est destiné à d'autres activités, telles comme la ferme ou terre inhabitée.

\section{CONCLUSIONS}

Les droits économiques, sociaux et culturels en Colombie se trouvent à un haut degré de développement malgré les multiples difficultés sociales et de budget du pays. La croissance de la jurisprudence constitutionnelle de la Cour Constitutionnelle a été formidable.

Le juge colombien est devenu un créateur et protecteur de droits sociaux, ce qui auparavant était une affaire exclusivement politique. II existe des conditions difficiles en Colombie pour obtenir une justice sociale efficace. La pauvreté, unie à l'isolement géographique de la population campagnarde, en plus des conflits armés internes, sont les conséquences de la intromission du juge constitutionnel dans la matérialisation de droits sociaux.

Toutefois, cet aspect sur les droits sociaux et leur protection conséquente par les juges au moyen d'actions constitutionnelles doit être mis en évidence. Cette position des juges a donné lieu à des confrontations avec le secteur politique qui ne voit pas d'un bon œil l'«interférence» de fonctionnaires d'une autre branche dans des affaires qui relevaient exclusivement de l'exécutif et du législatif. Mais la tâche protectrice de la Cour Constitutionnel colombien se poursuit.

\section{RÉFÉRENCES BIBLIOGRAPHIQUES}

ALEXY, R. (2002) Teoría de los derechos fundamentales. Madrid, Centro de Estudios Políticos y Constitucionales.

LANDA, C. (2002). Teoría de los derechos fundamentales. Dans: Cuestiones Constitucionales no. 6. Janvier - juin 2002, pag. 58.

VASAK, K. (2004). Les différentes typologies des droits de l'homme. Dans : Classer les droits de l'homme. Bruxelles.

\section{Jurisprudence}

CConst. C-1165/00, A. Beltrán.

CConst. C-220/11, E. Montealegre. 
CConst. C-228/11, J. Henao.

CConst. T-398/96, J. Arango.

CConst. T-283/06, A. Tafur

CConst. T-016/07, H. Sierra.

CConst. T-105/08, J. Córdoba

CConst. T-105/09, J. Córdoba

CConst. T-160/11 H. Sierra

CConst. T-1089/11, L. Vargas

CConst. T-749/12, M. Calle

CConst. T-567/12, M.Gonzalez

CConst. T-541/13, J. Pretelt

CConst. T-743/13L. Vargas 